\title{
FINITE-DIFFERENCE METHOD FOR PARAMETERIZED SINGULARLY PERTURBED PROBLEM
}

\author{
G. M. AMIRALIYEV, MUSTAFA KUDU, AND HAKKI DURU \\ Received 9 January 2004 and in revised form 22 April 2004
}

We study uniform finite-difference method for solving first-order singularly perturbed boundary value problem (BVP) depending on a parameter. Uniform error estimates in the discrete maximum norm are obtained for the numerical solution. Numerical results support the theoretical analysis.

\section{Introduction}

In this paper, we are going to devise a finite-difference method for the following parameter-dependant singularly perturbed boundary value problem (BVP):

$$
\begin{gathered}
L u:=\varepsilon u^{\prime}(x)+a(x) u(x)=f(x, \lambda), \quad x \in \Omega=(0, l], \\
u(0)=A, \quad u(l)=B,
\end{gathered}
$$

where $A, B$ are given constants and $a(x), f(x, \lambda)$ are sufficiently smooth functions such that

$$
\begin{gathered}
a(x) \geq \alpha>0 \quad \text { in } \bar{\Omega}=[0, l] \\
0<m_{1} \leq\left|\frac{\partial f}{\partial \lambda}\right| \leq M_{1}<\infty \quad \text { in } \bar{\Omega} \times \mathbb{R} .
\end{gathered}
$$

$\varepsilon>0$ is a small parameter and $\{u(x), \lambda\}$ is a solution.

For $\varepsilon \ll 1$, the function $u(x)$ has a boundary layer of thickness $O(\varepsilon)$ near $x=0$.

Under the above conditions, there exists a unique solution to problem (1.1), (1.2) (see $[7,12])$. An overview of some existence and uniqueness results and applications of parameterized equations may be obtained, for example, in $[6,7,8,9,12,13,15,16]$. In $[7,9,12]$, have also been considered some approximating aspects of this kind of problems. But designed in the above-mentioned papers, algorithms are only concerned with the regular cases (i.e., when the boundary layers are absent).

The numerical analysis of singular perturbation cases has always been far from trivial because of the boundary layer behavior of the solution. Such problems undergo rapid 
changes within very thin layers near the boundary or inside the problem domain $[4,10$, 11]. It is well known that standard numerical methods on uniform meshes for solving such problems are unstable and fail to give accurate results when the perturbation parameter $\varepsilon$ is small. Therefore, it is important to develop suitable numerical methods to these problems, whose accuracy does not depend on the parameter value $\varepsilon$, that is, methods that are convergent $\varepsilon$-uniformly. For the various approaches on the numerical solution of differential equations with steep, continuous solutions, we may refer to the monographs $[4,5,14]$.

Here we analyze a fitted difference scheme on a uniform mesh for the numerical solution of the problem (1.1), (1.2). In Section 2, we state some important properties of the exact solution. In Section 3, we present the difference scheme and obtain uniform error estimates for the truncation term and appropriate solution on a uniform mesh. Uniform convergence is proved in the discrete maximum norm. In Section 4, we formulate the iterative algorithm for solving the discrete problem and give the illustrative numerical results. The approach to construct discrete problem and error analysis for approximate solution is similar to those ones from $[1,2,3]$.

Henceforth, $C$ and $c$ denote the generic positive constants independent of $\varepsilon$ and of the mesh parameter. A subscripted such constant is also independent of $\varepsilon$ and mesh parameter, but whose value is fixed.

\section{The continuous problem}

In this section, we give uniform bounds of the solution of the BVP (1.1), (1.2), which will be used to analyze properties of the appropriate difference problem.

Lemma 2.1. For the solution $\{u(x), \lambda\}$ of the problem (1.1), (1.2),

$$
\begin{gathered}
|\lambda| \leq c_{0}, \\
\|u\|_{\infty} \leq c_{1},
\end{gathered}
$$

where

$$
\begin{gathered}
c_{0}=\frac{\|a\|_{\infty}}{m_{1}\left(1-\exp \left(-\|a\|_{\infty} l\right)\right)}(|A|+|B|)+m_{1}^{-1}\|F\|_{\infty}, \\
c_{1}=|A|+\alpha^{-1}\|F\|_{\infty}+c_{0} \alpha^{-1} M_{1}, \\
\left(F(x)=f(x, 0),\|a\|_{\infty} \equiv\|a\|_{\infty, \bar{\Omega}}:=\max _{\bar{\Omega}}|a(x)|\right) .
\end{gathered}
$$

Proof. We rewrite (1.1) as

$$
\varepsilon u^{\prime}(x)+a(x) u(x)=f(x, 0)+\frac{\partial \tilde{f}}{\partial \lambda} \lambda
$$

where

$$
\frac{\partial \tilde{f}}{\partial \lambda}=\frac{\partial f}{\partial \lambda}\left(x, \lambda^{*}\right), \quad \lambda^{*}=\gamma \lambda, 0<\gamma<1 .
$$


Integrating (2.4), we get

$$
\begin{aligned}
u(x)= & A \exp \left(-\frac{1}{\varepsilon} \int_{0}^{x} a(t) d t\right) \\
& +\frac{1}{\varepsilon} \int_{0}^{x} F(\tau) \exp \left(-\frac{1}{\varepsilon} \int_{\tau}^{x} a(t) d t\right) d \tau \\
& +\frac{1}{\varepsilon} \lambda \int_{0}^{x} \frac{\partial f}{\partial \lambda}\left(\tau, \lambda^{*}\right) \exp \left(-\frac{1}{\varepsilon} \int_{\tau}^{x} a(t) d t\right) d \tau,
\end{aligned}
$$

from which, by setting the boundary condition $u(l)=B$, we have

$$
\lambda=\frac{B-A \exp \left(-(1 / \varepsilon) \int_{0}^{l} a(t) d t\right)-(1 / \varepsilon) \int_{0}^{l} F(\tau) \exp \left(-(1 / \varepsilon) \int_{\tau}^{l} a(t) d t\right) d \tau}{(1 / \varepsilon) \int_{0}^{l}(\partial f / \partial \lambda)\left(\tau, \lambda^{*}\right) \exp \left(-(1 / \varepsilon) \int_{\tau}^{l} a(t) d t\right) d \tau} .
$$

Applying the mean-value theorem for integrals, we deduce that

$$
\begin{aligned}
& \frac{\left|(1 / \varepsilon) \int_{0}^{l} F(\tau) \exp \left(-(1 / \varepsilon) \int_{\tau}^{l} a(t) d t\right) d \tau\right|}{\left|(1 / \varepsilon) \int_{0}^{l}(\partial f / \partial \lambda)\left(\tau, \lambda^{*}\right) \exp \left(-(1 / \varepsilon) \int_{\tau}^{l} a(t) d t\right) d \tau\right|} \\
& \quad=\frac{\left|(1 / \varepsilon) \int_{0}^{l} F(\tau) \exp \left(-(1 / \varepsilon) \int_{\tau}^{l} a(t) d t\right) d \tau\right|}{(1 / \varepsilon)\left|(\partial f / \partial \lambda)\left(x^{*}, \lambda^{*}\right)\right| \int_{0}^{l} \exp \left(-(1 / \varepsilon) \int_{\tau}^{l} a(t) d t\right) d \tau} \\
& \leq m_{1}^{-1}\|F\|_{\infty}, \quad 0<x^{*}<l .
\end{aligned}
$$

It then follows from (2.7) that

$$
|\lambda| \leq \frac{\left|B-A \exp \left(-(1 / \varepsilon) \int_{0}^{l} a(t) d t\right)\right|}{m_{1}(1 / \varepsilon) \int_{0}^{l} \exp \left(-(1 / \varepsilon) \int_{\tau}^{l} a(t) d t\right) d \tau}+m_{1}^{-1}\|F\|_{\infty},
$$

which, for $\varepsilon \leq 1$, immediately leads to (2.1).

Next, from (2.6), we see that

$$
|u(x)| \leq|A| \exp \left(-\frac{\alpha x}{\varepsilon}\right)+\alpha^{-1}\left[1-\exp \left(-\frac{\alpha x}{\varepsilon}\right)\right]\left(\|F\|_{\infty}+|\lambda| M_{1}\right)
$$

and using the estimate (2.1), we obtain (2.2).

\section{Discrete problem and convergence}

3.1. Derivation of the difference scheme. In what follows, we denote by $\omega_{h}$ the uniform mesh on $\Omega$ :

$$
\omega_{h}=\left\{x_{i}=i h, i=1, \ldots, N ; N h=l\right\}, \quad \bar{\omega}_{h}=\omega_{h} \cup\{x=0\} .
$$




\section{Finite-difference method}

To simplify the notation, we set $g_{i}=g\left(x_{i}\right)$ for any function $g(x)$, while $g_{i}^{h}$ denotes an approximation of $g(x)$ at $x_{i}$.

For any mesh function $\left\{w_{i}\right\}$ defined on $\bar{\omega}_{h}$, we use the discrete maximum norm

$$
\|w\|_{\infty} \equiv\|w\|_{\infty, \bar{\omega}_{h}}:=\max _{0 \leq i \leq N}\left|w_{i}\right|
$$

The approach of generating difference method is through the integral identity

$$
\chi_{i} h^{-1} \int_{x_{i-1}}^{x_{i}} \operatorname{Lu} \varphi_{i}(x) d x=\chi_{i} h^{-1} \int_{x_{i-1}}^{x_{i}} f(x, \lambda) \varphi_{i}(x) d x, \quad 1 \leq i \leq N,
$$

with the exponential basis functions

$$
\varphi_{i}(x)=\exp \left(-\frac{a_{i}\left(x_{i}-x\right)}{\varepsilon}\right), \quad x_{i-1} \leq x \leq x_{i},
$$

where

$$
\chi_{i}=\left(h^{-1} \int_{x_{i-1}}^{x_{i}} \varphi_{i}(x) d x\right)^{-1}=\frac{a_{i} \rho}{1-\exp \left(-a_{i} \rho\right)}, \quad \rho=\frac{h}{\varepsilon}
$$

We note that function $\varphi_{i}(x)$ is the solution of the problem

$$
-\varepsilon \varphi_{i}^{\prime}(x)+a_{i} \varphi_{i}(x)=0, \quad x_{i-1} \leq x<x_{i}, \varphi_{i}\left(x_{i}\right)=1 .
$$

The relation (3.3) is rewritten as

$$
\chi_{i} h^{-1} \mathcal{\varepsilon} \int_{x_{i-1}}^{x_{i}} u^{\prime}(x) \varphi_{i}(x) d x+a_{i} \chi_{i} h^{-1} \int_{x_{i-1}}^{x_{i}} u(x) \varphi_{i}(x) d x+R_{i}=f\left(x_{i}, \lambda\right)
$$

with the remainder term

$$
\begin{aligned}
R_{i}= & \chi_{i} h^{-1} \int_{x_{i-1}}^{x_{i}}\left[a(x)-a\left(x_{i}\right)\right] \varphi_{i}(x) d x \\
& +\chi_{i} h^{-1} \int_{x_{i-1}}^{x_{i}}\left[f\left(x_{i}, \lambda\right)-f(x, \lambda)\right] \varphi_{i}(x) d x .
\end{aligned}
$$

To be consistent with $[1,2,3]$, we then obtain

$$
\varepsilon \theta_{i} u_{\bar{x}, i}+a_{i} u_{i}+R_{i}=f\left(x_{i}, \lambda\right), \quad 1 \leq i \leq N,
$$


where

$$
\begin{gathered}
\theta_{i}=1+\chi_{i} h^{-1} a_{i} \varepsilon^{-1} \int_{x_{i-1}}^{x_{i}}\left(x-x_{i}\right) \varphi_{i}(x) d x, \\
u_{\bar{x}, i}=\frac{u_{i}-u_{i-1}}{h},
\end{gathered}
$$

and a simple calculation gives us

$$
\theta_{i}=\frac{a_{i} \rho}{1-\exp \left(-a_{i} \rho\right)} \exp \left(-a_{i} \rho\right)
$$

As a consequence of (3.9), we propose the following difference scheme for approximating (1.1), (1.2):

$$
\begin{gathered}
L^{h} u_{i}^{h}:=\varepsilon \theta_{i} u_{\bar{x}, i}^{h}+a_{i} u_{i}^{h}=f\left(x_{i}, \lambda^{h}\right), \quad 1 \leq i \leq N, \\
u_{0}^{h}=A, \quad u_{N}^{h}=B,
\end{gathered}
$$

where $\theta_{i}$ is defined by (3.11).

3.2. Uniform error estimates. To investigate the convergence of the method, note that the error functions $z_{i}^{h}=u_{i}^{h}-u_{i}, 0 \leq i \leq N, \mu^{h}=\lambda^{h}-\lambda$ are the solution of the discrete problem

$$
\begin{gathered}
\varepsilon \theta_{i} z_{\bar{x}, i}^{h}+a_{i} z_{i}^{h}=f\left(x_{i}, \mu^{h}+\lambda\right)-f\left(x_{i}, \lambda\right)+R_{i}, \quad 1 \leq i \leq N, \\
z_{0}^{h}=0, \quad z_{N}^{h}=0,
\end{gathered}
$$

where $\theta_{i}$ and $R_{i}$ are given by (3.11) and (3.8), respectively.

Lemma 3.1. For the error function $R_{i}$,

$$
\|R\|_{\infty, \omega_{h}} \leq \mathrm{Ch},
$$

provided $a \in C^{1}(\bar{\Omega})$ and $|\partial f / \partial x| \leq C$ for $x \in \bar{\Omega}$ and $\lambda$ satisfying (2.1).

The proof easily follows from the explicit expression of $R_{i}$ defined by (3.8).

LEMma 3.2. The solution $\left\{z_{i}^{h}, \mu^{h}\right\}$ of the problem (3.13), (3.14) satisfies

$$
\begin{gathered}
\left|\mu^{h}\right| \leq m_{1}^{-1}\|R\|_{\infty, \omega_{h}}, \\
\left\|z^{h}\right\|_{\infty, \bar{\omega}_{h}} \leq \alpha^{-1}\left(1+m_{1}^{-1} M_{1}\right)\|R\|_{\infty, \omega_{h}} .
\end{gathered}
$$


Proof. From (3.13), we obtain

$$
z_{i}^{h}=\frac{\varepsilon \theta_{i}}{\varepsilon \theta_{i}+h a_{i}} z_{i-1}^{h}+\frac{h(\partial \tilde{f} / \partial \lambda)_{i}}{\varepsilon \theta_{i}+h a_{i}} \mu^{h}+\frac{h R_{i}}{\varepsilon \theta_{i}+h a_{i}},
$$

where

$$
\left(\frac{\partial \tilde{f}}{\partial \lambda}\right)_{i}=\frac{\partial f}{\partial \lambda}\left(x_{i}, \lambda+\gamma \mu^{h}\right), \quad 0<\gamma<1 .
$$

Solving the first-order difference equation with respect to $z_{i}^{h}$ and setting the boundary condition $z_{0}^{h}=0$, we get

$$
z_{i}^{h}=\mu^{h} h \sum_{k=1}^{i} \frac{(\partial \tilde{f} / \partial \lambda)_{k}}{\varepsilon \theta_{k}+h a_{k}} Q_{i k}+h \sum_{k=1}^{i} \frac{R_{k}}{\varepsilon \theta_{k}+h a_{k}} Q_{i k}
$$

where

$$
Q_{i k}= \begin{cases}1, & k=i, \\ \prod_{j=k+1}^{i} \frac{\varepsilon \theta_{j}}{\varepsilon \theta_{j}+h a_{j}}, & 1 \leq k \leq i-1 .\end{cases}
$$

For $i=N$, taking into consideration that $z_{N}^{h}=0$, we have

$$
\mu^{h}=-\frac{\sum_{k=1}^{N}\left(R_{k} /\left(\varepsilon \theta_{k}+h a_{k}\right)\right) Q_{N, k}}{\sum_{k=1}^{N}\left((\partial \tilde{f} / \partial \lambda)_{k} /\left(\varepsilon \theta_{k}+h a_{k}\right)\right) Q_{N, k}},
$$

from which, since $\varepsilon \theta_{i}+h a_{i}>0(1 \leq i \leq N)$, the required result (3.16) easily follows.

Finally, applying the maximum principle for difference operator $L^{h} z_{i}^{h}:=\varepsilon \theta_{i} z_{\bar{x}, i}^{h}+a_{i} z_{i}^{h}$, $1 \leq i \leq N$, to $(3.13)$ yields

$$
\left\|z^{h}\right\|_{\infty, \bar{\omega}_{h}} \leq \alpha^{-1}\left(M_{1}\left|\mu^{h}\right|+\|R\|_{\infty, \omega_{h}}\right),
$$

which, along with (3.16), leads to (3.17).

Combining the two previous lemmas gives us the following convergence result.

Theorem 3.3. Let $\{u(x), \lambda\}$ be the solution of (1.1), (1.2) and $\left\{u_{i}^{h}, \lambda^{h}\right\}$ the solution of (3.13), (3.14). Then

$$
\left|\lambda-\lambda^{h}\right| \leq C h, \quad\left\|u-u^{h}\right\|_{\infty, \bar{\omega}_{h}} \leq C h .
$$




\section{Numerical results}

In this section, we present some numerical experiments in order to illustrate the present method.

(a) We solve the nonlinear problem (3.12) using the following quasilinearization technique:

$$
\begin{gathered}
\varepsilon \theta_{i} u_{\tilde{x}, i}^{(n)}+a_{i} u_{i}^{(n)}=f\left(x_{i}, \lambda^{(n-1)}\right), \quad 1 \leq i<N, \\
u_{0}^{(n)}=A, \\
\lambda^{(n)}=\lambda^{(n-1)}-\frac{f\left(l, \lambda^{(n-1)}\right)-\theta_{N} \rho^{-1}\left(B-u_{N-1}^{(n)}\right)-a_{N} B}{(\partial f / \partial \lambda)\left(l, \lambda^{(n-1)}\right)},
\end{gathered}
$$

$n=1,2, \ldots ; \lambda^{(0)}$ given. (For simplicity, the $h$ on $u_{i}$ is omitted.) The initial guess $\lambda^{(0)}$ is being chosen by condition (2.1).

(b) Consider the test problem

$$
\begin{gathered}
\varepsilon u^{\prime}(x)+\frac{1}{1+x^{2}} u(x)=2 \lambda+\sin \frac{\lambda x}{2}, \quad 0 \leq x \leq 1, \\
u(0)=1, \quad u(1)=0 .
\end{gathered}
$$

The initial guess in (4.2) is taken as $\lambda^{(0)}=0.00039$ and stopping criterion is

$$
\max _{i}\left|u^{(n)}-u^{(n-1)}\right| \leq 10^{-5} ; \quad\left|\lambda^{(n)}-\lambda^{(n-1)}\right| \leq 10^{-5} .
$$

We use a double-mesh method (see, e.g., [5]) to compute the experimental rates of convergence:

$$
p_{u}^{\varepsilon, h}=\frac{\ln \left(e_{u}^{\varepsilon, h} / e_{u}^{\varepsilon, h / 2}\right)}{\ln 2}, \quad e_{u}^{\varepsilon, h}=\max _{0 \leq i \leq N}\left|u_{i}^{h}-u_{2 i}^{h / 2}\right|
$$

for $u_{i}^{h}$, and

$$
p_{\lambda}^{\varepsilon, h}=\frac{\ln \left(e_{\lambda}^{\varepsilon, h} / e_{\lambda}^{\varepsilon, h / 2}\right)}{\ln 2}, \quad e_{\lambda}^{\varepsilon, h}=\left|\lambda^{h}-\lambda^{h / 2}\right|
$$

for $\lambda^{h}$.

Tables 4.1 and 4.2 contain some numerical results for different values of $\varepsilon$ and $h$, based on the double-mesh principle. The result established here is that the discrete solution is uniformly convergent with respect to the perturbation parameter, and also clearly, that we obtain first-order convergence, so Theorem 3.3 is sharp. 
Table 4.1. Errors $\left\{e_{u}^{\varepsilon, h}, e_{\lambda}^{\varepsilon, h}\right\}$ and convergence rates $\left\{p_{u}^{\varepsilon, h}, p_{\lambda}^{\varepsilon, h}\right\}$ on $\omega_{h}$ for $h=1 / 8$ and $h=1 / 16$.

\begin{tabular}{c|cc|cc|cc}
\hline \multirow{2}{*}{$\varepsilon$} & \multicolumn{2}{|c|}{$h=1 / 8$} & \multicolumn{2}{c|}{$h=1 / 16$} & \multirow{2}{*}{$p_{u}^{\varepsilon, h}$} & $p_{\lambda}^{\varepsilon, h}$ \\
\cline { 2 - 5 } & $e_{u}^{\varepsilon, h}$ & $e_{\lambda}^{\varepsilon, h}$ & $e_{u}^{\varepsilon, h / 2}$ & $e_{\lambda}^{\varepsilon, h / 2}$ & & \\
\hline $10^{-3}$ & 0.00002235 & 0.00000506 & 0.00001069 & 0.00000248 & 1.064 & 1.028 \\
$10^{-4}$ & 0.00002235 & 0.00000506 & 0.00001069 & 0.00000248 & 1.064 & 1.027 \\
$10^{-5}$ & 0.00002235 & 0.00000506 & 0.00001069 & 0.00000248 & 1.064 & 1.027 \\
$10^{-6}$ & 0.00002235 & 0.00000506 & 0.00001069 & 0.00000248 & 1.064 & 1.027 \\
$10^{-7}$ & 0.00002235 & 0.00000506 & 0.00001069 & 0.00000248 & 1.064 & 1.027 \\
$10^{-8}$ & 0.00002235 & 0.00000506 & 0.00001069 & 0.00000248 & 1.064 & 1.027 \\
$10^{-9}$ & 0.00002235 & 0.00000506 & 0.00001069 & 0.00000248 & 1.064 & 1.027 \\
$10^{-10}$ & 0.00002235 & 0.00000506 & 0.00001069 & 0.00000248 & 1.064 & 1.027 \\
$10^{-11}$ & 0.00002235 & 0.00000506 & 0.00001069 & 0.00000248 & 1.064 & 1.027 \\
$10^{-12}$ & 0.00002235 & 0.00000506 & 0.00001069 & 0.00000248 & 1.064 & 1.027 \\
\hline
\end{tabular}

Table 4.2. Errors $\left\{e_{u}^{\varepsilon, h}, e_{\lambda}^{\varepsilon, h}\right\}$ and convergence rates $\left\{p_{u}^{\varepsilon, h}, p_{\lambda}^{\varepsilon, h}\right\}$ on $\omega_{h}$ for $h=1 / 16$ and $h=1 / 32$.

\begin{tabular}{c|cc|cc|cc}
\hline \multirow{2}{*}{$\varepsilon$} & \multicolumn{2}{|c|}{$h=1 / 16$} & \multicolumn{2}{c|}{$h=1 / 32$} & \multirow{2}{*}{$p_{u}^{\varepsilon, h}$} & \multirow{2}{*}{$p_{\lambda}^{\varepsilon, h}$} \\
\cline { 2 - 5 } & $e_{u}^{\varepsilon, h}$ & $e_{\lambda}^{\varepsilon, h}$ & $e_{u}^{\varepsilon, h / 2}$ & $e_{\lambda}^{\varepsilon, h / 2}$ & & \\
\hline $10^{-3}$ & 0.00001152 & 0.00000248 & 0.000000566 & 0.00000122 & 1.028 & 1.028 \\
$10^{-4}$ & 0.00001152 & 0.00000248 & 0.00000571 & 0.00000123 & 1.014 & 1.014 \\
$10^{-5}$ & 0.00001152 & 0.00000248 & 0.00000571 & 0.00000123 & 1.014 & 1.014 \\
$10^{-6}$ & 0.00001152 & 0.00000248 & 0.00000571 & 0.00000123 & 1.014 & 1.014 \\
$10^{-7}$ & 0.00001152 & 0.00000248 & 0.00000571 & 0.00000123 & 1.014 & 1.014 \\
$10^{-8}$ & 0.00001152 & 0.00000248 & 0.00000571 & 0.00000123 & 1.014 & 1.014 \\
$10^{-9}$ & 0.00001152 & 0.00000248 & 0.00000571 & 0.00000123 & 1.014 & 1.014 \\
$10^{-10}$ & 0.00001152 & 0.00000248 & 0.00000571 & 0.00000123 & 1.014 & 1.014 \\
$10^{-11}$ & 0.00001152 & 0.00000248 & 0.00000571 & 0.00000123 & 1.014 & 1.014 \\
$10^{-12}$ & 0.00001152 & 0.00000248 & 0.00000571 & 0.00000123 & 1.014 & 1.014 \\
\hline
\end{tabular}

\section{Acknowledgment}

The authors would like to thank the referees for their very useful comments and suggestions.

\section{References}

[1] G. M. Amiraliyev, Difference method for the solution of one problem of the theory dispersive waves, Differ. Uravn. 26 (1990), 2146-2154 (Russian).

[2] G. M. Amiraliyev and H. Duru, A uniformly convergent finite difference method for a singularly perturbed initial value problem, Appl. Math. Mech. (English Ed.) 20 (1999), no. 4, 379-387.

[3] _ A uniformly convergent difference method for the periodical boundary value problem, Comput. Math. Appl. 46 (2003), no. 5-6, 695-703.

[4] E. P. Doolan, J. J. H. Miller, and W. H. A. Schilders, Uniform Numerical Methods for Problems with Initial and Boundary Layers, Boole Press, Dún Laoghaire, 1980. 
[5] P. A. Farrell, A. F. Hegarty, J. J. H. Miller, E. O’Riordan, and G. I. Shishkin, Robust Computational Techniques for Boundary Layers, Applied Mathematics (Boca Raton), vol. 16, Chapman \& Hall/CRC, Florida, 2000.

[6] M. Fečkan, Parametrized singularly perturbed boundary value problems, J. Math. Anal. Appl. 188 (1994), no. 2, 426-435.

[7] A. Gulle and H. Duru, Convergence of the iterative processes to the solution of the boundary problem with the parameter, Trans. Acad. Sci. Azerb. Ser. Phys.-Tech. Math. Sci. Math. Mech. 18 (1998), no. 3-4, 34-40.

[8] T. Jankowski, Monotone iterations for differential problems, Math. Notes (Miskolc) 2 (2001), no. $1,31-38$.

[9] T. Jankowski and V. Lakshmikantham, Monotone iterations for differential equations with a parameter, J. Appl. Math. Stochastic Anal. 10 (1997), no. 3, 273-278.

[10] A. H. Nayfeh, Introduction to Perturbation Techniques, John Wiley \& Sons, New York, 1981.

[11] R. E. O’Malley Jr., Singular Perturbation Methods for Ordinary Differential Equations, Applied Mathematical Sciences, vol. 89, Springer-Verlag, New York, 1991.

[12] T. Pomentale, A constructive theorem of existence and uniqueness for the problem $y^{\prime}=f(x, y, \lambda)$, $y(a)=\alpha, y(b)=\beta$, Z. Angew. Math. Mech. 56 (1976), no. 8, 387-388.

[13] M. Rontó and T. Csikos-Marinets, On the investigation of some non-linear boundary value problems with parameters, Math. Notes (Miskolc) 1 (2000), no. 2, 157-166.

[14] H.-G. Roos, M. Stynes, and L. Tobiska, Numerical Methods for Singularly Perturbed Differential Equations, Springer Series in Computational Mathematics, vol. 24, Springer-Verlag, Berlin, 1996.

[15] S. Staněk, Nonlinear boundary value problem for second order differential equations depending on a parameter, Math. Slovaca 47 (1997), no. 4, 439-449.

[16] K. Zawischa, Uber die Differentialgleichung deren Losungskurve durch zwei gegebene Punkte hindurchgehen soll, Monatsh. Math. 37 (1930), 103-124 (German).

G. M. Amiraliyev: Department of Mathematics, Faculty of Science, Yuzuncu Yil University, 65080 Van, Turkey

E-mail address: gamirali2000@yahoo.com

Mustafa Kudu: Department of Mathematics, Faculty of Education, Dicle University, Siirt, 21280 Diyarbakir, Turkey

E-mail address: muskud28@yahoo.com

Hakki Duru: Department of Mathematics, Faculty of Science, Yuzuncu Yil University, 65080 Van, Turkey

E-mail address: hakkiduru2002@yahoo.com 


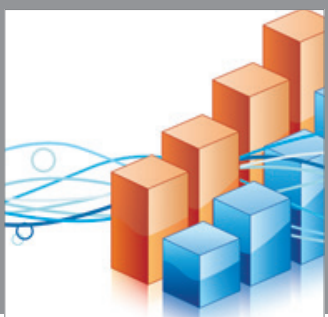

Advances in

Operations Research

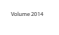

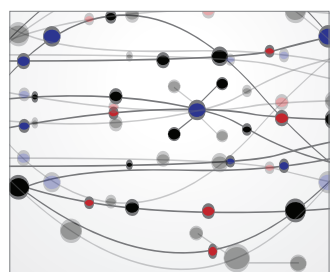

\section{The Scientific} World Journal
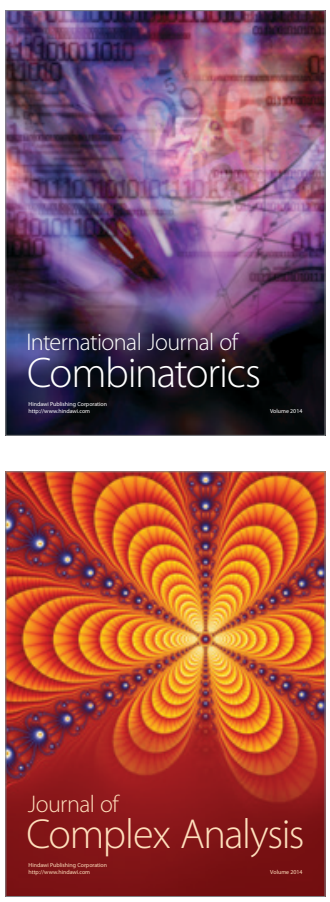

International Journal of

Mathematics and

Mathematical

Sciences
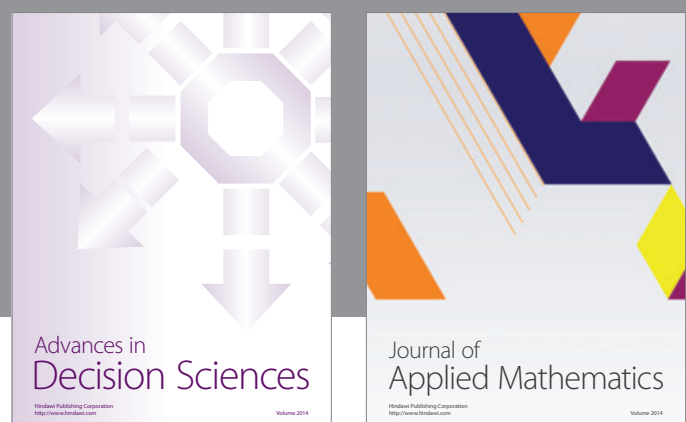

Journal of

Applied Mathematics
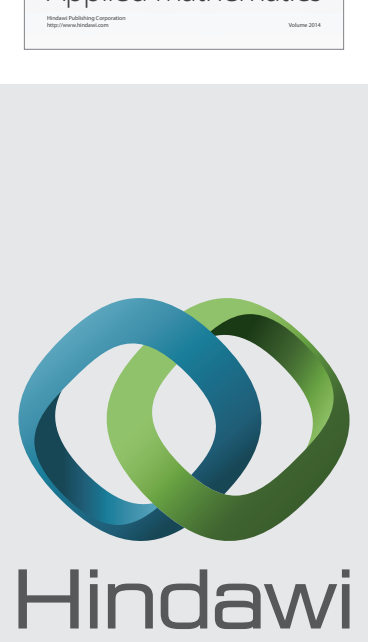

Submit your manuscripts at http://www.hindawi.com
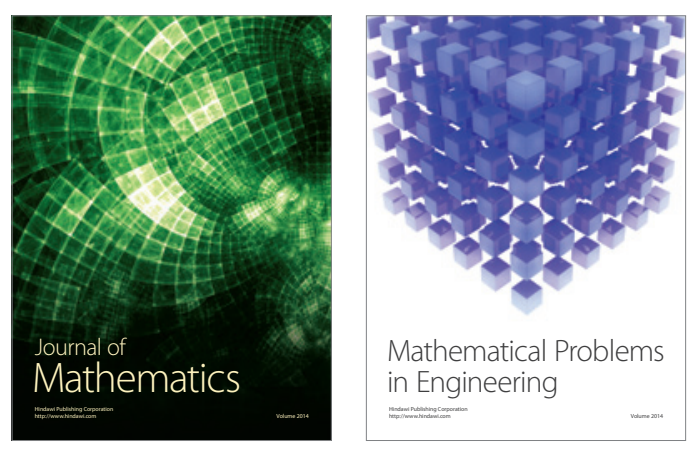

Mathematical Problems in Engineering
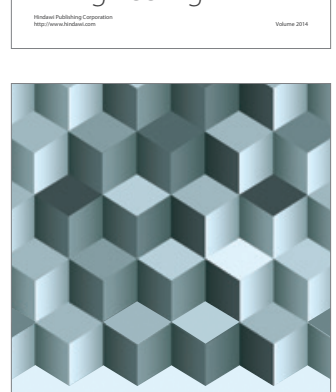

Journal of

Function Spaces
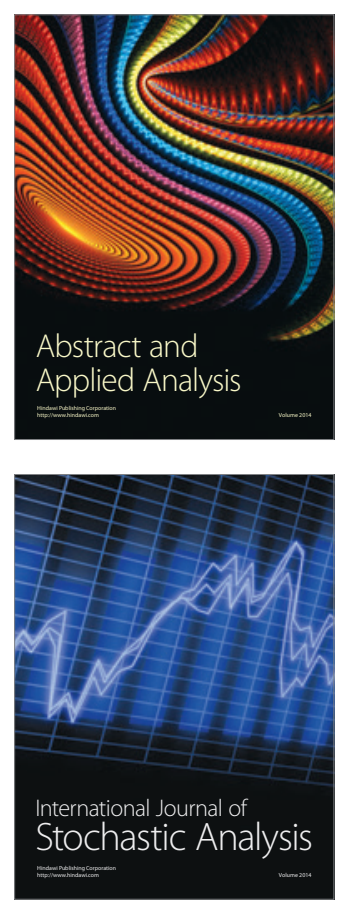

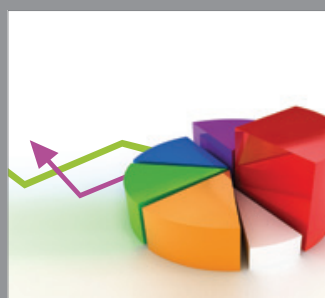

ournal of

Probability and Statistics

Promensencen
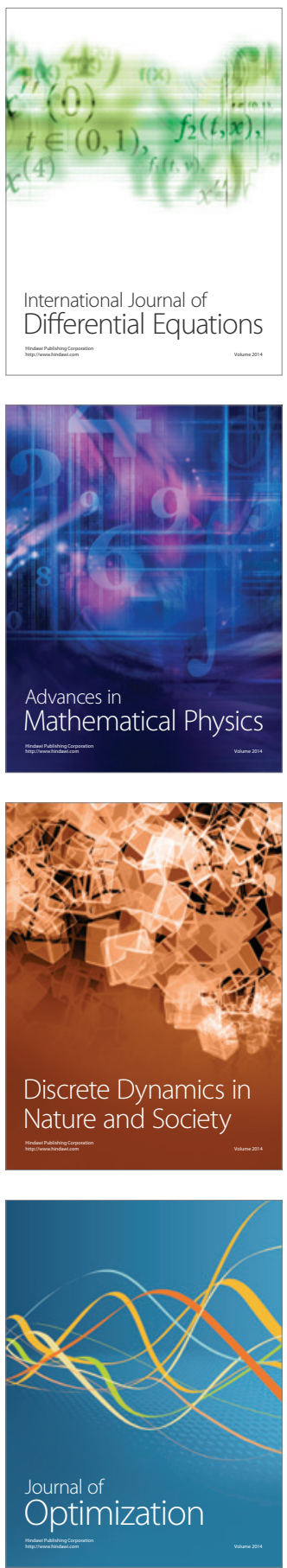